Cite this: Phys. Chem. Chem. Phys., 2012, 14, 11633-11638

\title{
Quantifying barriers to monovalent anion transport in narrow non-polar pores
}

\author{
Laura A. Richards, ${ }^{a b}$ Andrea I. Schäfer, ${ }^{b}$ Bryce S. Richards ${ }^{a}$ and Ben Corry ${ }^{* c}$ \\ Received 21st May 2012, Accepted 26th June 2012 \\ DOI: $10.1039 / \mathrm{c} 2 \mathrm{cp} 41641 \mathrm{~g}$
}

The transport of anionic drinking water contaminants (fluoride, chloride, nitrate and nitrite) through narrow pores ranging in effective radius from 2.5 to $6.5 \AA$ was systematically evaluated using molecular dynamics simulations to elucidate the magnitude and origin of energetic barriers encountered in nanofiltration. Free energy profiles for ion transport through the pores show that energy barriers depend on pore size and ion properties and that there are three key regimes that affect transport. The first is where the ion can fit in the pore with its full inner hydration shell, the second is where the pore size is between the bare ion and hydrated radius, and the third is where the ion size approaches that of the pore. Energy barriers in the first regime are relatively small and due to rearrangement of the inner hydration shell and/or displacement of further hydration shells. Energy barriers in the second regime are due to partial dehydration and are larger than barriers seen in the first regime. In the third regime, the pore becomes too small for bare ions to fit regardless of hydration and thus energy barriers are very high. In the second regime where partial dehydration controls transport, the trend in the slopes of the change in energy barrier with pore size corresponds to the hydration strength of the anions.

\section{Introduction}

Understanding water and ion transport through confined pores is crucially important because of applications such as ion removal by nanofiltration membranes, ${ }^{1,2}$ transport in carbon nanotubes ${ }^{3,4}$ and understanding biological ion channels. ${ }^{5}$ In all of these processes, ions permeate through confined channels in either a hydrated, dehydrated or partially hydrated state. However, despite its importance, the role of hydration in determining ion transport through narrow pores remains poorly understood and quantifying the effect of partial dehydration is difficult. Significant effort has been made to understand the hydration of cations during transport through ion channels ${ }^{6-13}$ and chloride and cations during transport in nanopores, ${ }^{14,15}$ however this has not yet been thoroughly extended to anionic contaminants that are particularly important for drinking water applications. A recent study demonstrated that the cost of partial dehydration is the largest contribution to the barriers determining transport of anions through narrow, cylindrical non-polar pores (which are simplified compared to real nanofiltration "pores"). ${ }^{16}$ However, a thorough analysis of the barriers seen by each anion (fluoride, chloride, nitrate and nitrite) at a range of pore sizes was

\footnotetext{
${ }^{a}$ School of Engineering and Physical Sciences, Heriot-Watt University, Edinburgh, EH14 4AS, United Kingdom

${ }^{b}$ School of Engineering, The University of Edinburgh, Edinburgh, EH9 3JL, United Kingdom

${ }^{c}$ Research School of Biology, Australian National University, Canberra, ACT 0200, Australia.E-mail: ben.corry@anu.edu.au
}

not conducted. Gaining an appreciation of how the hydration state of anions influences their retention by nanofiltration membranes will be important for developing new membranes that can remove these contaminants more efficiently. Ion hydration has also been suggested to play a role in creating the anion selectivity seen in a range of biological anion channels that contain narrow non-polar pores, as there appears to be a relation between ion conductivity and hydration strength. ${ }^{17,18}$ However, the effect of needing to only partial dehydrate anions to enter the pores has not been adequately considered in explaining anion selectivity. This current study endeavors to address these issues by quantifying the influence of hydration of four anion types as they permeate through pores of a range of sizes by evaluating energetic barriers of transport for each scenario.

\section{Methods}

The barriers to ion transport in narrow pores were determined here using classical molecular dynamics (MD) simulations employing previously determined force fields for fluoride and chloride ${ }^{19}$ and those specifically developed for nitrate $\left(\mathrm{NO}_{3}{ }^{-}\right)$ and nitrite $\left(\mathrm{NO}_{2}{ }^{-}\right) \cdot{ }^{16} \mathrm{MD}$ simulations were conducted by the authors using the model we previously described in detail. ${ }^{16}$ The software programs utilized were NAMD2. $7^{20}$ for simulation and VMD1.9 $9^{21}$ for visualization. Each simulation contained a single cylindrical pore, which was represented as a smooth, rigid, un-charged, non-polar idealized surface to be generic and avoid 
specific chemical characteristics. Closely spaced discrete atoms were used to represent the surface, spaced at an interval of $1 \AA$ and with Lennard-Jones interactions defined by the interaction distance $\left(r_{\text {min,surface }}=3.75 \AA\right)$ and potential energy well depth $\left(\varepsilon_{\text {surface }}=0.1946 \mathrm{kcal} \mathrm{mol}^{-1}\right)$, based upon values for methyl groups in hydrocarbon chains. ${ }^{22,23}$ The pore was solvated in a non-polarizable TIP3 $\mathrm{P}^{24,25}$ water box of dimensions $40 \times 40 \times 70 \AA$ with periodic boundary conditions for continuity. All simulations were ionized for a net concentration of $0.1 \mathrm{M}$ single sodium salt (note that the pore holds only one ion at a time). Fluoride, chloride, nitrate and nitrite were selected as they are all monovalent anionic drinking water contaminants. Temperature and pressure were controlled using Langevin dynamics and a Langevin piston, with the damping coefficient for temperature control set at $5 \mathrm{ps}^{-1}$ and the oscillation period for pressure control set at $200 \mathrm{ps}$. Full-system periodic electrostatics (Ewald summation) were applied. In order to represent the space available to water in the pore, an effective pore radius $\left(R_{\text {eff }}\right)$ was determined by calculating the oxygen density profile of water within the pore, and adding the distance at which it became zero to the ionic radius of oxygen $\left(R_{\text {ion,oxygen }}=1.77 \AA\right)$ in water. Pore radii were selected to be similar to nanofiltration membranes and narrow carbon nanotubes and ranged from 2.5 to $6.5 \AA$. A $16 \AA$ pore length was selected for computational efficiency as increasing the pore length had almost no effect on the barriers to ion transport, since the barriers in such pores have been shown to arise at the pore entrance and exit. ${ }^{26}$ While a longer pore does slightly affect the quantification of energy barriers due to the electric field of the ion, the impact of energy barrier is small $(<10 \%$ difference between a $16 \AA$ and $32 \AA$ pore $)$ and has no effect on selectivity trends. Hydrated radius was defined as the first minima in the ion-oxygen radial distribution function found in bulk water, and the coordination number was defined as the average number of water molecules within the hydrated radius.

The free energy (potential of mean force) of each ion passing through the pore was determined using umbrella sampling ${ }^{27}$ in which a harmonic potential was used to set the location of the ion of interest along a trajectory path defined by the distance along the pore axis $(Z)$ and distance from this axis $\left(r_{\text {radial }}\right) .1 \AA$ steps were used from $Z=-15 \AA$ (bulk) to $0 \AA$ (center of pore). Additional positions were sampled from $Z=-15$ to $7 \AA$ (at $r_{\text {radial }}=4 \AA$ ) for effective pore radius $R_{\text {eff }}=2.8$ and $3.3 \AA ; Z=-15$ to $0 \AA$ (at $r_{\text {radial }}=4 \AA$ ) for $R_{\text {eff }}=3.7$ and $4.3 \AA$; and $Z=-15$ to $0 \AA$ (at $r_{\text {radial }}=4 \AA$ and $8 \AA$ ) for $R_{\text {eff }}=$ 5.3 and $6.5 \AA$. The applied force constants were $2 \mathrm{kcal} \mathrm{mol}^{-1} \AA^{-2}$ and $0.2 \mathrm{kcal} \mathrm{mol}^{-1} \AA^{-2}$ in the $Z$ and $r_{\text {radial }}$ directions, respectively. Target positions and applied force constants were selected to ensure complete sampling. A 250 ps simulation was run at each target position. The weighted histogram analysis method (WHAM) ${ }^{28,29}$ was used to calculate two-dimensional free energy profiles with a tolerance of 0.0001 and 30 bins in both $Z$ and $r_{\text {radial }}$ directions. Two-dimensional profiles were integrated at each $Z$ position ${ }^{26}$ to determine a one-dimensional profile. All energy profiles were single-ion profiles as no other ions entered the pores during the simulations. Reproducibility was determined to be $\pm 2.3 \%$ from the standard deviation of the peak of the energy barrier of seven independent trajectories of fluoride for $R_{\mathrm{eff}}=3.3 \AA$.

\section{Results and discussion}

The magnitude of the energy barriers faced by each ion is plotted versus the effective pore size in Fig. 1. This figure shows that energy barriers for fluoride, chloride, nitrate and nitrite are a clear function of pore size. This figure shows additional information not described in our earlier work, ${ }^{16}$ specifically results for four ion types rather than only fluoride. This additional information shows that there appear to be three key regimes for each ion with regard to the magnitude of the energy barriers. These key regimes describe situations in which:

1. the ion fits in pore with complete inner hydration shell $\left(R_{\mathrm{hyd}}<R_{\mathrm{eff}}\right)$;

2. the pore size is between the size of the bare ion and hydrated ion $\left(R_{\text {ion }}<R_{\text {eff }}<R_{\text {hyd }}\right)$;

3. the bare ion does not fit inside the pore $\left(R_{\mathrm{ion}}>R_{\mathrm{eff}}\right)$.

In Regime 1 , the effective pore size is larger than the hydrated radius of the ion of interest. This is the regime the farthest on the right in Fig. 1A-D. In this case, since the hydrated ion can fit in the pore, stripping of the first water shell will not occur during transport and thus the hydrated ion passes through the pore unhindered and unretained. Energy barriers in this regime are relatively small and are due to rearrangement/reorientation of the first hydration shell or loss of the second and more distant hydration shell. The increase in energy barriers with decreasing pore size, even when the ion can fit in the pore with its hydrated shell, is most significant for strongly hydrated fluoride and chloride.

In Regime 2, some dehydration is required in order for the ion to enter the pore with a partial hydration shell. Pore size significantly impacts the energy barriers in this regime due to the number of water molecules that need to be removed from the ion and the associated energy required to partially dehydrate the ion. As water molecules are stripped away from the ion, the ion holds the remaining water molecules more tightly, which explains the steep increase in slope. This can be seen in Regime 2 in Fig. 1A-D and is related to each ion's hydration energy. Fluoride, the most strongly hydrated, has a very steep slope in this regime (starting immediately from the transition from Regime 1 to Regime 2), followed by chloride, nitrite and nitrate. This trend in slope is the same as the trend in absolute values of hydration energies, where fluoride $\left(-119.7 \mathrm{kcal} \mathrm{mol}^{-1}\right)^{30}>$ chloride $\left(-89.1 \mathrm{kcal} \mathrm{mol}^{-1}\right)^{30}>$ nitrite $\left(-78.8 \mathrm{kcal} \mathrm{mol}^{-1}\right)^{31}>$ nitrate $\left(-71.6 \mathrm{kcal} \mathrm{mol}^{-1}\right){ }^{31}$ At the farthest left of Regime 2, the slopes of the barriers of all ions increase substantially due to the strong attraction to the last remaining water molecules, even for relatively weakly hydrated nitrate and nitrite.

In Regime 3, energy barriers become extremely high, as eventually the ion will no longer fit inside the pore. In our results, fluoride and chloride never reached this third regime because their small ionic size (similar to that of water) was smaller than the smallest pore size studied. Both nitrate and nitrite entered Regime 3 for $R_{\text {eff }}=2.5$ and $2.8 \AA$. Here energy barriers are much higher than obtained in larger pore sizes for nitrate and nitrite $\left(>35 \mathrm{kcal} \mathrm{mol}^{-1}\right)$, due to the bare ions being too large to fit in the pore, and thus transport through the pore would be very unlikely. Nitrite does not experience as steep an increase in energy barrier below $R_{\text {eff }}=2.8 \AA$ as nitrate, even though the bare ion size is larger than the effective 


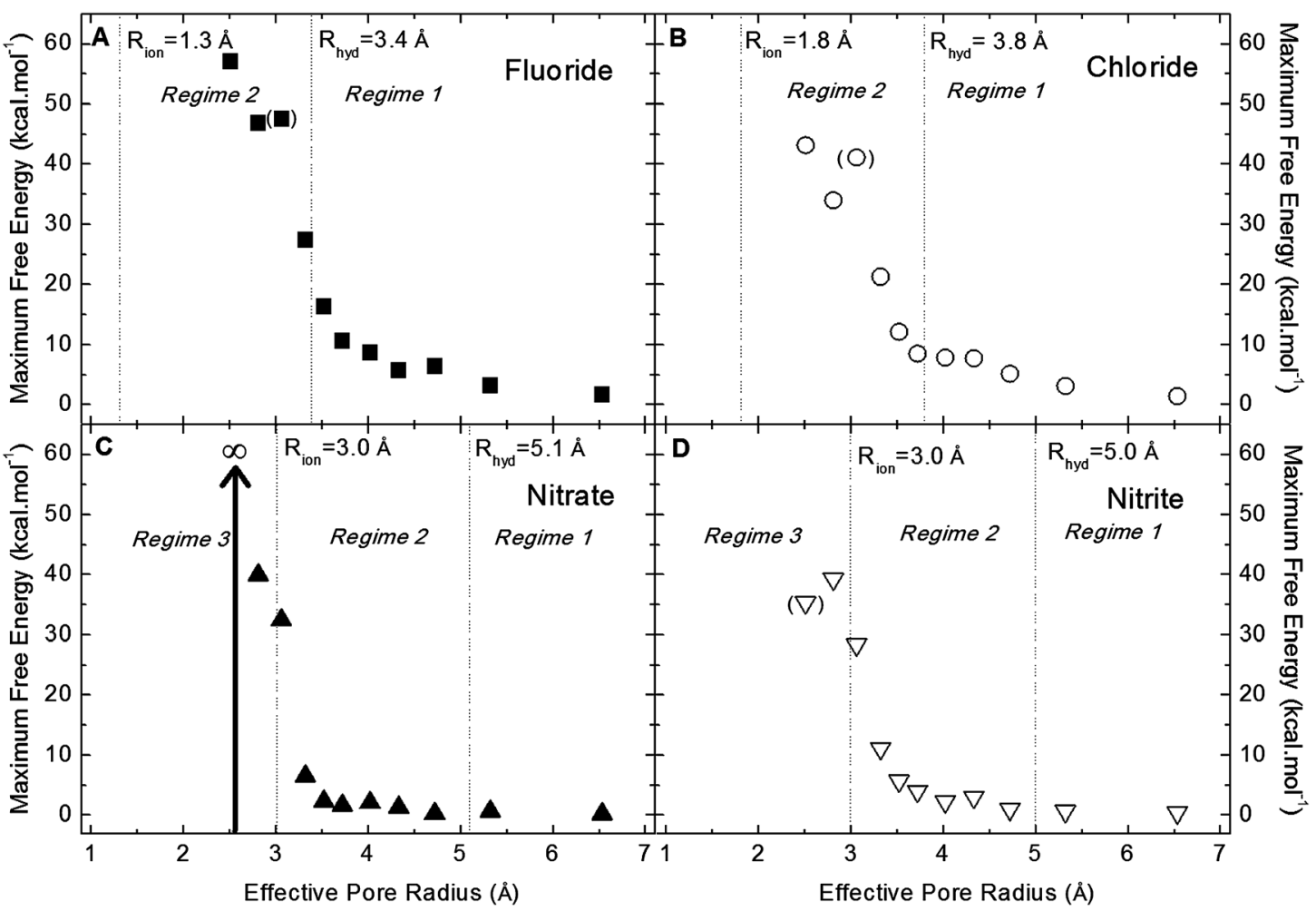

Fig. 1 Maximum energy barriers for (A) fluoride, (B) chloride, (C) nitrate and (D) nitrite passing through the pore for a range of pore sizes.

pore radius. This can be explained by considering the different shapes of nitrite and nitrate and their possible orientations in the pore. Although we have defined the bare ion radius of nitrite to be the same as nitrate $(3.0 \AA)$ based on the nitrogen-oxygen distance, this does not account for the different shapes of the molecules. While both molecules are planar, nitrite only has two oxygen atoms, and is thus more linear than nitrate, allowing it to orient such that it fits more easily into a narrow pore.

The order of the energy barriers seen by the different ions, or "selectivity sequence" to use the language of ion channels, changes according to the pore size since the regimes for each of the ions are located according to the different ion properties (ion size, hydrated size, hydration strength). This ordering is shown on Table 1. The ordering corresponds to which regime the specific ion is at for a particular pore size, and thus the ordering is directly related to the amount of partial dehydration required. Because each of the ions has a different ionic size, hydrated size and hydration strength, the selectivity sequence will change depending on pore radius. This phenomenon was previously demonstrated for fluoride and chloride, ${ }^{16}$ where there was a swap in sequence from $R_{\text {eff }}=3.3 \AA$ to $4.3 \AA$ due to fluoride being sufficiently small to fit into the $4.3 \AA$ pore radius with its first hydration shell complete, whereas chloride still needed to partially dehydrate, leading to a higher barrier for chloride than fluoride. This is different than in the smaller $3.3 \AA$ pore radius, where fluoride had a higher barrier than chloride because both ions faced partial dehydration but the stronger hydration free energy of fluoride meant that the stripping of water molecules carried a higher energy expense (and thus energy barrier).

This same concept explains the changes in selectivity sequences for all ions and all pore sizes presented in Table 1. At the smallest
Table 1 Energy barriers determined for each ion at each pore radius. The standard deviation of the peak of the energy barrier for seven independent, identical trajectories is $\pm 2.3 \%$ and this value is assumed to be similar for each trajectory. Nitrite data at $2.5 \AA$ is assumed to be out of trend (as shown on Fig. 1)

\begin{tabular}{ll}
\hline \multirow{2}{*}{$\begin{array}{l}\text { Effective pore } \\
\text { radius }(\AA)\end{array}$} & Energy barrier $\left(\mathrm{kcal} \mathrm{mol}^{-1}\right)$ \\
\hline 2.5 & $\mathrm{NO}_{3}^{-}(\infty)>\mathrm{F}^{-}(57.1)>\mathrm{NO}_{2}^{-}(35.3)>\mathrm{Cl}^{-}(43.2)$ \\
2.8 & $\mathrm{~F}^{-}(46.9)>\mathrm{NO}_{3}^{-}(40.0)>\mathrm{NO}_{2}^{-}(39.3)>\mathrm{Cl}^{-}(34.0)$ \\
3.1 & $\mathrm{~F}^{-}(47.7)>\mathrm{Cl}^{-}(41.1)>\mathrm{NO}_{3}^{-}(32.5)>\mathrm{NO}_{2}^{-}(28.4)$ \\
3.3 & $\mathrm{~F}^{-}(27.4)>\mathrm{Cl}^{-}(21.3)>\mathrm{NO}_{2}^{-}(11.1)>\mathrm{NO}_{3}^{-}(6.5)$ \\
3.5 & $\mathrm{~F}^{-}(16.4)>\mathrm{Cl}^{-}(12.1)>\mathrm{NO}_{2}^{-}(5.7)>\mathrm{NO}_{3}{ }^{-}(2.3)$ \\
3.7 & $\mathrm{~F}^{-}(10.6)>\mathrm{Cl}^{-}(8.5)>\mathrm{NO}_{2}{ }^{-}(4.0)>\mathrm{NO}_{3}^{-}(1.6)$ \\
4.0 & $\mathrm{~F}^{-}(8.6)>\mathrm{Cl}^{-}(7.8)>\mathrm{NO}_{2}{ }^{-}(2.2) \approx \mathrm{NO}_{3}^{-}(2.1)$ \\
4.3 & $\mathrm{Cl}^{-}(7.7)>\mathrm{F}^{-}(5.7)>\mathrm{NO}_{2}^{-}(3.0)>\mathrm{NO}_{3}^{-}(1.3)$ \\
4.7 & $\mathrm{~F}^{-}(6.4)>\mathrm{Cl}^{-}(5.2)>\mathrm{NO}_{2}^{-}(1.0)>\mathrm{NO}_{3}^{-}(0.3)$ \\
5.3 & $\mathrm{~F}^{-}(3.3) \approx \mathrm{Cl}^{-}(3.1)>\mathrm{NO}_{2}^{-}(0.7) \approx \mathrm{NO}_{3}^{-}(0.7)$ \\
6.5 & $\mathrm{~F}^{-}(1.7) \approx \mathrm{Cl}^{-}(1.4)>\mathrm{NO}_{2}^{-}(0.4) \approx \mathrm{NO}_{3}^{-}(0.2)$ \\
\hline
\end{tabular}

pore size $\left(R_{\mathrm{eff}}=2.5 \AA\right)$, the energy barriers are nitrate $>$ nitrite $>$ fluoride $>$ chloride, assuming the nitrite data point is out of trend. Here, nitrate and nitrite are too large to fit in the pore at all, resulting in very high barriers for these ions. At $2.8 \AA$, the order changes to fluoride $>$ nitrate $>$ nitrite $>$ chloride, because nitrate and nitrite can now fit in but the dehydration requirements for fluoride are extremely high leading to fluoride having the highest barrier. At $3.1 \AA$, ordering is fluoride $>$ chloride $>$ nitrate $>$ nitrite, as all are in the second regime and hence some dehydration is required (with the energy barriers resulting in differences in hydration free energy). From $3.3 \AA$ to $4.0 \AA$, the order is fluoride $>$ chloride $>$ nitrite $>$ nitrate, which is the inverse 
order of the hydrated radii. At $4.3 \AA$, the order is chloride > fluoride $>$ nitrite $>$ nitrate, due to fluoride fitting in with a complete hydration shell whereas chloride still required partial dehydration. Finally at $4.7 \AA$ to $6.5 \AA$, the order is fluoride $>$ chloride $>$ nitrite $=$ nitrate, where each ion is able to fit in with its first hydration shell intact (first regime) but barriers still result from the displacement of more distant water for strongly hydrated fluoride and chloride.

Because of the idealized construction of the simplified pore, energy barriers obtained in this work cannot be directly compared to those obtained in biological channels or nanofiltration membranes due to considerations such as polarity, functional groups, tortuousity and pore size distributions. Despite these limitations in making a direct comparison, the energy barriers obtained are very reasonable with those obtained in other studies. For examples, the energy barrier of chloride transport in a non-polar, 3.0 $\mathrm{A}$ radius, closed state acetylcholine receptor is approximately $4 \mathrm{kcal} \mathrm{mol}^{-1} \cdot{ }^{32,33} \mathrm{In}$ nanofiltration membranes, energy barriers to salt transport evaluated experimentally with the Arrhenius relationship have been reported in the range of 2.3-12.9 $\mathrm{kcal} \mathrm{mol}^{-1}$. $^{34-37}$ Conventionally, selectivity in nanofiltration is evaluated using retention. The general ordering of the retention of these salts is fluoride $>$ chloride $>$ nitrate $>$ nitrite. ${ }^{37-42}$

To better understand the origin and magnitude of the energy barriers and confirm that the barriers obtained are due to dehydration, the coordination numbers of each ion for each pore size are plotted. Fig. 2A shows average coordination number in the center of the pore versus effective pore radii. For the smallest pore sizes, where energy barriers are the highest, fluoride and chloride have a single chain of water molecules on either side, making the coordination number limited to two inside the pore. As the pore size increases, more water molecules are able to fit inside the pore with the ions in all cases. At pore sizes larger than the hydrated radii of the ion, the coordination number does not change as the maximum number of water molecules are associated with the ion, independent of being in the bulk water or in the pore.

Fig. 2B shows the direct link between the energy barriers and the dehydration required for each ion to enter the pore. At the smallest pore sizes, where the most dehydration is required, the ratio of the coordination number in the pore to coordination number in bulk is the lowest, and consequentially the energy barrier is the highest. The steeper increase in barrier as the coordination number pore to bulk ratio decreases is because the ion holds on to the last remaining water molecules more strongly. This is the case for all ions evaluated, although the actual values of course depend on the ion properties. The close relationship between the energy barrier and degree of dehydration confirms that the energy barriers obtained are due to varying degrees of dehydration.

A plot of the average interaction energy of each ion with the water molecules within its first hydration shell further confirms that barriers are due to dehydration (Fig. 2C). As pore size increases, the magnitude of the attractive (negative) interaction increases as more water surrounds each ion. The interaction energies reach approximately the same value as in bulk at different pore sizes according to the ion properties. Importantly, the interaction of the ion with water alone for different pore
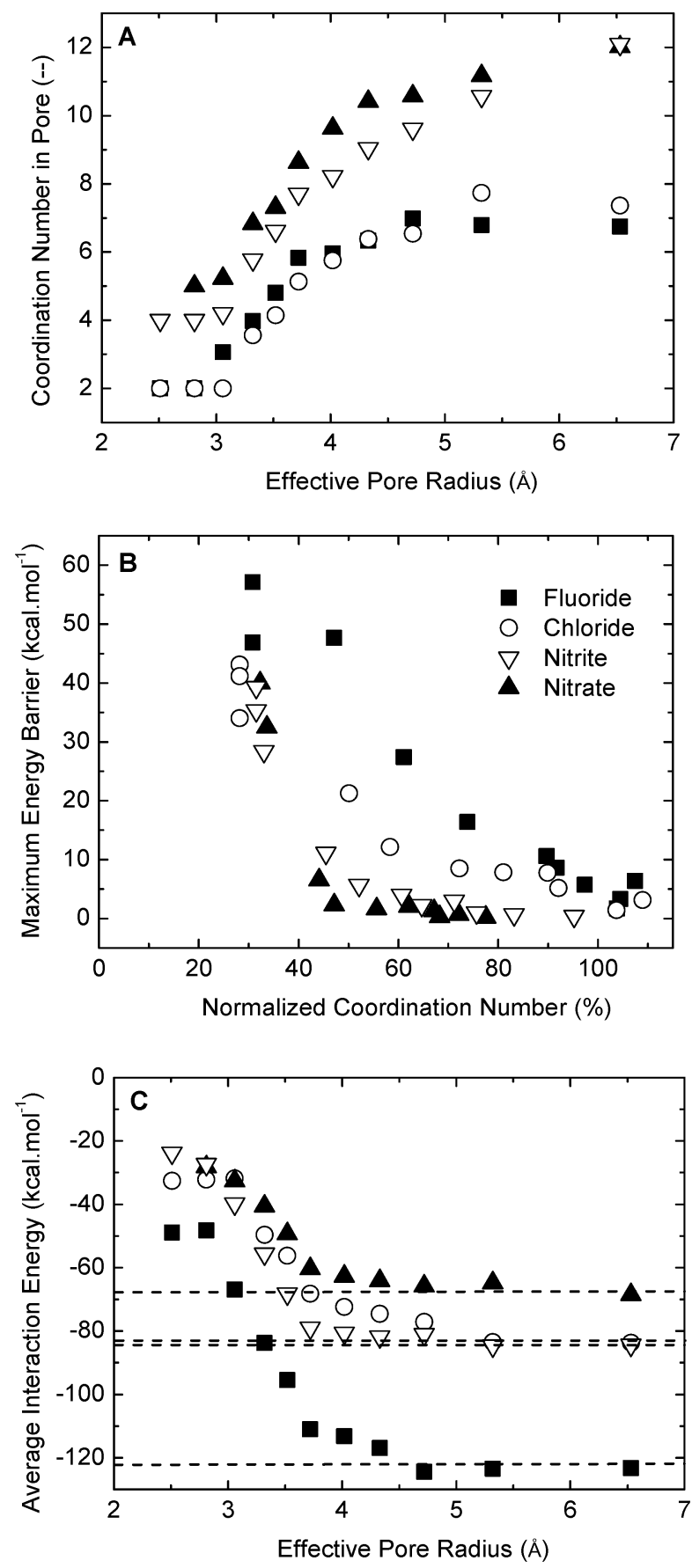

Fig. 2 Relating energy barriers to ion hydration. (A) Ion coordination numbers in pore center versus effective pore radius $\left(R_{\mathrm{eff}}\right)$; (B) Maximum energy barrier versus normalized coordination number (average coordination number in pore divided by average coordination number in bulk); (C) Average interaction energy of each ion with its first hydration shell when located in pore center (dashed line indicates interaction energy in bulk). The standard deviation of average interaction energy in bulk $(n=11)$ is determined to be for fluoride $\pm 0.8 \%$; chloride $\pm 0.6 \%$; nitrite $\pm 1.9 \%$; nitrate $\pm 3.2 \%$.

sizes follows the same trend as the total free energy change, with the magnitude being similar to the hydration free energies. As these results are average interaction energies, they do not include entropic contributions and thus cannot be directly compared 

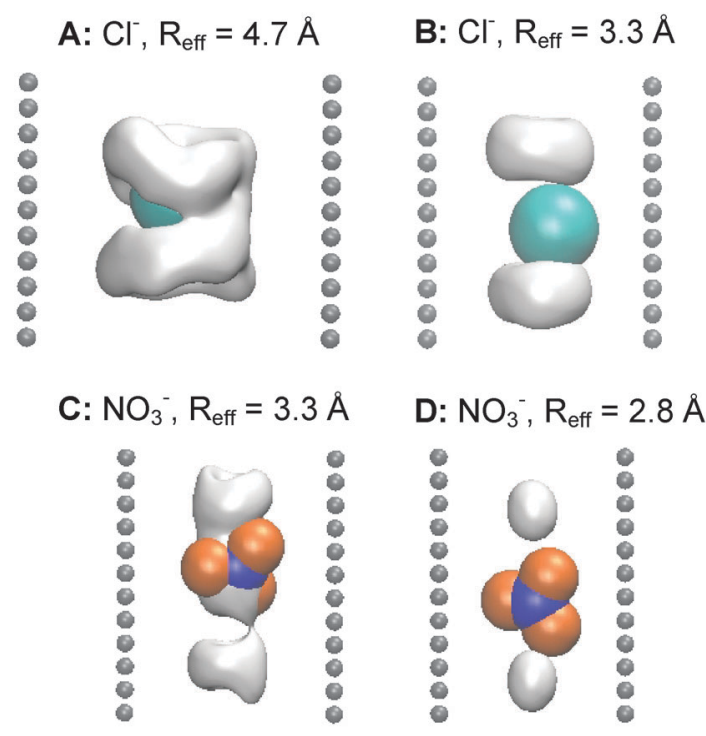

Fig. 3 Structure of water around the selected ion at the center of a pore as represented by isosurface plots of the average water density in the hydrated radius. (A) chloride at $R_{\mathrm{eff}}=4.7 \AA$ (Regime 1); (B) chloride at $R_{\text {eff }}=3.3 \AA$ (Regime 2); (C) nitrate at $R_{\text {eff }}=3.3 \AA$ (Regime 2); (D) nitrate at $R_{\text {eff }}=2.8 \AA$ (Regime 3).

to the free energy barriers (free energy barriers include both enthalpic and entropic contributions), however, they do support that dehydration is the major cause of the free energy barriers. Further, a comparison with partial dehydration energies for chloride previously reported ${ }^{43}$ shows a similar trend and magnitude to the results obtained here, again supporting the claim that the obtained energy barriers are due to partial dehydration.

Finally, the effect of partial ion dehydration is demonstrated visually using a probability density function of water within the hydrated radius around each ion in the center of the pore (Fig. 3). Each of the regimes can be seen here. The first regime is shown in Fig. 3A where the water surrounds the chloride ion in the pore. The second regime is shown for chloride and nitrate (Fig. 3B and C, respectively), where the ion must be partially dehydrated to enter the pore (e.g. $\left.R_{\mathrm{ion}}<R_{\mathrm{eff}}<R_{\mathrm{hyd}}\right)$ and water forms a single file chain on either side of the ion. The final regime, where it is difficult for the bare ion to fit, is shown in Fig. 3D. Here the area that the water in the pore fills is smaller than the bare nitrate ion, highlighting the unfavorable nature of this regime.

\section{Conclusions}

In conclusion, by calculating the energetics of transport of a number of anions through narrow, cylindrical, un-charged, non-polar pores, we have shown that energy barriers for ion permeation are a function of ion type and pore size. Energy barriers show three regimes with respect to pore size. These regimes describe regimes in which (1) an ion fits in a pore with its complete hydration shell; (2) the pore size is between the bare and hydrated ion such that partial dehydration is required; (3) the bare ion is too large to fit in the pore. Because each regime occurs at different pore sizes for the different ions, the ordering of barrier heights (selectivity sequence) changes with pore size. While a narrow pore hinders the large ions most strongly due to direct discrimination based upon size, intermediate pores block the most strongly hydrated ions, and the larger pores again can hinder the larger ions as the smaller ions can pass with a complete hydration shell. The models described here involve simplified non-polar pores with limited interactions between the ion and the pore walls. In these circumstances the energy barriers seen by ions as they pass through the pores are largely due to the cost of dehydrating the ion. It is of interest in future work to evaluate dehydration barriers to multi-charged and/or more chemically advanced drinking water contaminants, such as sulfates and boron, and to systematically adjust pore properties. The inclusion of polar groups in the pore walls will lead to additional interactions that can add to or compensate for the dehydration affects described here. Nanofiltration membranes, for example, are often made of polyamide materials with polar groups that can interact with the ion which may reduce the barriers to ion transport. These results described here are significant because they improve the understanding of the mechanistic role of hydration as an important factor in determining anion transport in narrow pores. Careful tailoring of the pore properties may enable these dehydration barriers to be harnessed to improve the rejection of anionic contaminants in nanofiltration and thus can contribute to future membrane models and design. Comparison of the results obtained here with the atomic resolution structures of biological anion channels may also allow for an explanation of ion selectivity in these narrow non-polar pores.

\section{Acknowledgements}

The authors would like to thank IChemE, Joint Research Institute in Energy (Heriot-Watt University), the School of Engineering and Physical Sciences (Heriot-Watt University), Overseas Research Students Awards Scheme and James Watt Scholarship for financial support for Laura Richards' PhD studentship and visit to the University of Western Australia. All computation work was done using a grant under the merit allocation scheme of the National Computational Infrastructure (NCI) National Facility (Australia) and additional computer time from iVEC.

\section{References}

1 W. R. Bowen and J. S. Welfoot, Modelling the performance of membrane nanofiltration - critical assessment and model development, Chem. Eng. Sci., 2002, 57, 1121-1137.

2 B. Tansel, J. Sager, T. Rector, J. Garland, R. Strayer, L. Lanfang, M. Roberts, M. Hummerick and J. Bauer, Significance of hydrated radius and hydration shells on ionic permeability during nanofiltration in dead end and cross flow modes, Sep. Purif. Technol., 2006, 51, 40-47.

3 B. J. Hinds, N. Chopra, T. Rantell, R. Andrews, V. Gavalas and L. G. Bachas, Aligned multiwalled carbon nanotube membranes, Science, 2004, 303, 62-65.

4 K. P. Lee, T. C. Arnot and D. Mattia, A review of reverse osmosis membrane materials for desalination - Development to date and future potential, J. Membr. Sci., 2011, 370, 1-22.

5 M. Ashcroft, From molecule to malady, Nature, 2006, 440, 440-447.

6 A. Miyazawa, Y. Fujiyoshi and N. Unwin, Structure and gating mechanisms of the acetylcholine receptor pore, Nature, 2003, 423, 949-955. 
7 Y. Zhou, J. H. Morais-Cabral, A. Kaufman and R. MacKinnon, Chemistry of ion coordination and hydration revealed by a $\mathrm{K}+$ channel-Fab complex at $2.0 \AA$ resolution, Nature, 2001, 414, 43-48.

8 O. Beckstein, K. Tai and M. S. P. Sansom, Not ions alone: Barriers to ion permeation in nanopores and channels, J. Am. Chem. Soc., 2004, 126, 14694-14695.

9 S. Varma and S. B. Rempe, Coordination numbers of alkali metal ions in aqueous solutions, Biophys. Chem., 2006, 124, 192-199.

10 D. T. Edmonds, Membrane ion channels and ionic hydration energies, Proc. R. Soc. London, Ser. B, 1980, 211, 51-62.

11 M. Carrillo-Tripp, M. L. San-Román, J. Hernańdez-Cobos, H. Saint-Martin and I. Ortega-Blake, Ion hydration in nanopores and the molecular basis of selectivity, Biophys. Chem., 2006, 124, 243-250.

12 B. Hille, The Hydration of Sodium Ions Crossing the Nerve Membrane, Proc. Natl. Acad. Sci. U. S. A., 1971, 68, 280-282.

$13 \mathrm{~S}$. Y. Noskov and B. Roux, Importance of Hydration and Dynamics on the Selectivity of the KcsA and NaK Channels, J. Gen. Physiol., 2007, 129, 135-143.

14 M. Zwolak, J. Lagerqvist and M. Di Ventra, Quantized Ionic Conductance in Nanopores, Phys. Rev. Lett., 2009, 103, 128102-128106.

15 M. Zwolak, J. Wilson and M. D. Ventra, Dehydration and ionic conductance quantization in nanopores, J. Phys.: Condens. Matter, 2010, 22, 454126.

16 L. A. Richards, A. I. Schäfer, B. S. Richards and B. Corry, The importance of dehydration in determining ion transport in narrow pores, Small, 2012, 8, 1701-1709.

17 Y. Chen, L. Hu, M. Punta, R. Bruni, B. Hillerich, B. Kloss, B. Rost, J. Love, S. A. Siegelbaum and W. A. Hendrickson, Homologue structure of the SLAC1 anion channel for closing stomata in leaves, Nature, 2010, 467, 1074-1080.

18 B. K. Czyzewski and D. N. Wang, Identification and characterization of a bacterial hydrosulphide ion channel, Nature, 2012, 483, 494- 497.

19 I. S. Joung and T. E. Cheatham III, Determination of alkali and halide monovalent ion parameters for use in explicitly solvated biomolecular simulations, J. Phys. Chem. B, 2008, 112, 9020-9041.

20 J. C. Phillips, R. Braun, W. Wang, J. Gumbart, E. Tajkhorshid, E. Villa, C. Chipot, R. B. Skeel, L. Kalé and K. Schulten, Scalable molecular dynamics with NAMD, J. Comput. Chem., 2005, 26, 1781-1802.

21 W. Humphrey, A. Dalke and K. Schulten, VMD - Visual Molecular Dynamics, J. Mol. Graphics, 1996, 14, 33-38.

22 R. Allen, J. P. Hansen and S. Melchionna, Molecular dynamics investigation of water permeation through nanopores, J. Chem. Phys., 2003, 119, 3905-3919.

23 M. G. Martin and J. I. Siepmann, Transferable potentials for phase equilibria. 1. United-atom description of $n$-alkanes, J. Phys. Chem. B, 1998, 102, 2569-2577.

24 W. L. Jorgensen, J. Chandrasekhar and J. D. Madura, Comparison of simple potential functions for simulating liquid water, J. Chem. Phys., 1983, 79, 926-935.

25 O. M. Becker, A. D. M. Jr, B. Roux and M. Watanabe, Computational Biochemistry and Biophysics, Marcel Dekker, New York, 2001.
26 B. Corry, Designing carbon nanotube membranes for efficient water desalination, J. Phys. Chem. B, 2008, 112, 1427-1434.

27 G. M. Torrie and J. P. Valleau, Monte Carlo free energy estimates using non-Boltzmann sampling: Application to the sub-critical Lennard-Jones fluid, Chem. Phys. Lett., 1974, 28, 578-581.

28 S. Kumar, J. M. Rosenberg, D. Bouzida, R. H. Swendsen and P. A. Kollman, The weighted hisogram analysis method for freeenergy calculations on biomolecules. I. The method, J. Comput. Chem., 1992, 13, 1011-1021.

29 B. Roux, The calculation of the potential of mean force using computer simulations, Comput. Phys. Commun., 1995, 91, 275-282.

30 R. Schmid, A. M. Miah and V. N. Sapunov, A new table of the thermodynamic quantities of ionic hydration: values and some applications (enthalpy-entropy compensation and Born radii), Phys. Chem. Chem. Phys., 2000, 2, 97-102.

31 Y. Marcus, Thermodynamics of solvation of ions. Part 5: Gibbs free energy of hydration at 298.15 K, J. Chem. Soc., Faraday Trans., 1991, 87, 2995-2999.

32 O. Beckstein and M. S. Sansom, A hydrophobic gate in an ion channel: the closed state of the nicotinic acetylcholine receptor, Phys. Biol., 2006, 3, 147-159.

33 J. Cohen and K. Schulten, Mechanism of Anionic Conduction across ClC, Biophys. J., 2004, 86, 836-845.

34 J. Y. Chen, H. Nomura and G. G. Wade, Pusch, Temperature dependence of membrane transport parameters in hyperfiltration, Desalination, 1983, 46, 437-446.

35 V. Freger, T. C. Arnot and J. A. Howell, Separation of concentrated organic/inorganic salt mixtures by nanofiltration, J. Membr. Sci., 2000, 178, 185-193.

36 H. H. Kim, J. H. Kim and Y. K. Chang, Removal of potassium chloride by nanofiltration from ion-exchanged solution containing potassium clavulanate, Bioprocess Biosyst. Eng., 2010, 33, 149-158.

37 R. R. Sharma and S. Chellam, Temperature and concentration effects on electrolyte transport across porous thin-film composite nanofiltration membranes: Pore transport mechanisms and energetics of permeation, J. Colloid Interface Sci., 2006, 298, 327-340.

38 A. Lhassani, M. Rumeau, D. Benjelloun and M. Pontie, Selective Demineralization of Water by Nanofiltration Application to the Defluorination of Brackish Water, Water Res., 2001, 35, 3260-3264.

39 L. Paugam, C. K. Diawara, J. P. Schlumpf, P. Jaouen and F. Quéméneur, Transfer of monovalent anions and nitrates especially through nanofiltration membranes in brackish water conditions, Sep. Purif. Technol., 2004, 40, 237-242.

40 L. Paugam, S. Taha, G. Dorange, P. Jaouen and F. Quéméneur, Mechanism of nitrate ions transfer in nanofiltration depending on pressure, $\mathrm{pH}$, concentration and medium concentration, J. Membr. Sci., 2004, 231, 37-46.

41 L. A. Richards, M. Vuachère and A. I. Schäfer, Impactof pH on the removal of fluoride, nitrate and boron by nanofiltration/reverse osmosis, Desalination, 2010, 261, 331-337.

42 B. Van der Bruggen, A. Koninckx and C. Vandecasteele, Separation of monovalent and divalent ions from aqueous solution by electrodialysis and nanofiltration, Water Res., 2004, 38, 1347-1353.

43 C. Song and B. Corry, Intrinsic ion selectivity of narrow hydrophobic pores, J. Phys. Chem. B, 2009, 113, 7642-7649. 